\title{
5 Noch krank? Selber schuld! Gesundheit 2040.
}

\author{
Björn Zeien
}

Das Gesundheitswesen. Wir schreiben das Jahr 2040. Unbekannte Welten warten auf uns. Oder wissen wir nicht bereits, was auf uns zukommen wird?

\section{Der Gesundheitscheck: Jeden Tag zu jeder Zeit}

Ein persönliches Hologramm begrüßt uns morgens mit neuen Nachrichten und Anrufen sowie To-dos und Terminen des beginnenden Tages. Bei Nutzung der Toilette, beim Rasieren oder Duschen erfolgen Check-ups: Infekte, Vital- und Körperwerte, Spuren von Abszessen, Neurodermitis, Pilzerkrankungen, Flechten, Gürtelrose, Karzinomen, Gen-Mutationen oder drohendes Organversagen werden direkt festgestellt. Bei letzterem erfolgt das Aktivieren des Selbstheilungsprozesses von erkrankten Zellen oder die Herstellung neuer Organe aus eigenen Stammzellen - ohne eigenes Zutun. Weitere Auffälligkeiten werden durch Algorithmen bewertet, gegebenenfalls wird ein Arzt hinzugezogen. Als Hologramm bei Routinebefunden, per Video-Sprechstunde, wenn ein „echter“ Arzt benötigt wird. Bei medikamentösen Behandlungen werden Wirkstoffpatronen per Drohne zugestellt. Eingesetzt in den persönlichen 3D-Drucker folgt die Tablettenherstellung. Wirkstoffe, Nanotechnik und Dosierungen sind speziell auf das eigene Erbgut abgestimmt. Die Einnahme wird über Gesten protokoliert, die Abrechnung erfolgt einnahmegerecht, der personalisierte Medikationsplan ergänzt sich automatisch. Die Tablette findet selbstständig zu dem Wirkungsort und entfaltet über die Neuausrichtung der Nanopartikel Ihre Wirkung. Überschüssige Wirkstoffe werden über Körperausscheidungen festgestellt und haben Auswirkungen auf die nachfolgende individualisierte Tablettenherstellung. Neben- 
wirkungen reduzieren, Wirkungsweise und Therapieerfolg erhöhen sich. Krankheitswahrscheinlichkeiten bei ausbleibender oder falscher Behandlung zeigen persönliche Gesundheitsstatistiken an. Der Einfluss des Lebenswandels bzw. von Behandlungen lassen Rückschlüsse auf die eigene Gesunderhaltung zu.

Ein Schreckensszenario oder die Aussicht für eine neue gesellschaftliche Ausrichtung?

\section{Eine neue Kultur: Die Gesundheitsgesellschaft}

Im Jahr 2040 wird der heute als zweiter Gesundheitsmarkt bekannte Bereich eng verschmolzen mit der Regelversorgung sein. Alternative Heilmethoden zusammen mit der Schulmedizin ergeben eine neue Versorgungsvielfalt. Die Gesundheit ist ein Lebensgefühl. Ernährung, Sport - oder anders-, der Körper selbst bestimmt dabei die Lebensqualität. Dies nimmt einen bisher nicht gekannten Stellenwert in der Gesellschaft ein. Ein vielseitiger Nachfragemarkt für innovative Gesundheitsdienstleistungen gemixt aus lokalen, regionalen und bundesweiten Angeboten entsteht. Patientenindividuelle Leistungen pro Behandlungsfeld und Mensch zur Erreichung des bestmöglichen Ergebnisses sind selbstverständlich. Ein Arzt wird lange vor einer Erkrankung aufgesucht.

Aber was genau bedeutet dies? Der Schlüssel für eine immer älter werdende Gesellschaft liegt in der körperlichen und geistigen Gesunderhaltung. Die Gesundheit selbst wird zum Konsumgut und lässt die Menschen gesünder und somit zufriedener durch ein längeres Leben gehen. Angebote rund um das Thema Gesundheit ergeben einen Zukunftsmarkt mit unvorstellbaren Wachstumschancen. Die Vision heißt: Man betrachtet nur einen Prozess. Das Leben! Jeder möchte diesen „Prozess“ möglichst lange und gesund durchlaufen. Wenn dieser Prozess bereinigt um die behandelnden Akteure betrachtet werden würde, könnten grundsätzlich neue Angebote entstehen. Beim Sport fällt eine Kurzatmigkeit zusammen mit Knieschmerzen auf. Das Angebot: Einmal Herzaufbau, neues Kniegelenk aus der Retorte und Reha-Maßnahmen. Nach intensiver ineinandergreifender Behandlung wird der Patient entlassen. Ohne Schmerzen, ohne Probleme beim Sport.

Das Gesundheitswesen selbst wird patientenorientierter. Im Jahr 2040 entwickelt sich der mündige Patient zum gesundheitsbewussten Individualisten, erhält ,by the way" Informationen aus medizinischen Diagnose-Tools auf bildgebende Kontaktlinsen. Diese Informationen ermächtigen ihn zur gesundheitlichen Eigenverantwortung, auch um sein persönliches Wohlbefinden mitzugestalten. Sein umfangreiches Gesundheitswissen führt zu Patientengesprächen auf Augenhöhe und zugleich zur Einigung auf gemeinsame Behandlungsmethoden. Konsumverhalten überträgt sich auf Gesundheitsleistungen, Vergleiche dieser bzw. von Ärzten, Kliniken und Dienstleistern unter Preis- und Qualitätskriterien sind selbstverständlich. Die Kultur der aktiven Mitgestaltung entwickelt sich zu einer Gesundheitsgesellschaft. Der Patient erhält Einblicke in Dokumentationen seiner Behandlungen, Laborwerte, Kosten- und Erstattungsstrukturen. Gesundheit „to go“ ergeben neue Angebote: Der Urlaub zum Ausbau der Gesundheit inklusive operativer Eingriffe oder vorbeugender Therapien dient der patientenindividuellen Gesunderhaltung eines jeden Einzelnen. Private Anbieter stellen Angebote für Luxuskonsumenten. Gesundheitsversorgung erhält 
Einzug in das 5-Sterne-Segment. Im noblen Ambiente werden beispielsweise individualisierte Gentherapien angeboten. Der Behandlungserfolg nimmt Einfluss auf die Bepreisung. Erst bei Erfolg wird bezahlt.

\section{Vernetzung kennt weder Landes- noch Sektorengrenzen}

Weiterentwicklungen von Informations- und Kommunikationstechnologien führen zur grundlegenden digitalen Vernetzung innerhalb des gesamten Gesundheitswesens. Wearables, elektronische Tattoos und intelligente Kleidung ausgestattet mit Sensoren geben Vitalwerte im Jahr 2040 an die Systeme des „Ambient Assisted Living“ oder „Smart Home“ weiter, Hilfsmittel informieren Dienstleister über Anomalien (beispielsweise Sturz, zu geringe Flüssigkeitsaufnahme, ...). Bei Nutzen erfolgt die Intervention zusammen mit dem Arzt, kognitive Systeme mit künstlicher Intelligenz übernehmen die Steuerung im häuslichen Umfeld und sorgen somit für eine positive Einflussnahme auf den Verlauf patientenindividualisierter Heilungsprozesse. Zusatzinformationen, eingeblendet in die reale Welt (Augmented Reality) unterstützen dabei den Menschen. Verlaufsinformation von Behandlungen durch Systeme im häuslichen Umfeld wird an behandelnde Akteure weitergegeben. Die Vernetzung führt zu verkürzten Vorbereitungszeiten auf medizinischer Seite, Behandlungsempfehlungen wiederum werden im häuslichen Umfeld überwacht. Der Mensch selbst steht im Zentrum der digitalen Vernetzung.

\section{Große Datenmengen, großer Nutzen}

Anonymisierte bzw. pseudonymisierte Gesundheitsdaten nehmen Einfluss auf die Diagnosestellung. Sämtliche am Patienten handelnde Personen wie Ärzte, Apotheker, Gesundheitsfachberufler und weitere Dienstleister dokumentieren ihre fachlichen, Patienten ihre sportlichen und freizeitlichen Aktivitäten in ineinandergreifenden Portalen oder mithilfe von Wearables. Ein bisher unbekanntes Volumen von großen Datenmengen entsteht. Diese stehen der Forschung zur Verfügung. Virtuelle Medikamentenerprobungen ersetzen aufwendige Studien mit Patientenbeteiligung. Wirkstoffkombinationen werden in kürzester Zeit erprobt. Die Ergebnisse führen zu neuen Erkenntnissen, Gesundheitsdienstleistungen werden angepasst. Seltene Erkrankungen werden ausfindig gemacht, vorausschauende Prävention zusammen mit ganzheitlichen Versorgungskonzepten zur Gesunderhaltung haben kürzere Behandlungendauern und erhöhte Behandlungserfolge zur Folge. Jeder Patient profitiert, die Lebensqualität steigt, die Gesundheitskosten sinken.

\section{Fazit: Fiction oder Future?}

Zur Erinnerung: Am 8. September 1966 erfolgte die Ausstrahlung der ersten Folge der Serie „Star Trek“ in den USA. Kaum einer ahnte damals, dass der Kommunikator, die Sprachsteuerung von Computern oder gar tragbare Computer einzig über Bildschirme steuerbar irgendwann einmal Realität werden würden. Aber was bedeutet dies für unser Gesundheitssystem? 
Die aus heutiger Sicht zukunftsorientierten Ambient-Assisted-Living- und Personal Health- Systemlösungen werden 2040 den Alltag begleiten. Sie kommunizieren mit tragbaren medizinischen Geräten für den Hausgebrauch, unterstützen den Menschen bei der Anwendung, beeinflussen Behandlungsverläufe positiv und stehen dabei im Austausch mit dem begleitenden Mediziner. Die Interaktion von Mensch und Technik führt zur dezentralen Visualisierung von medizinischen Gesundheitsdienstleistungen. Die gesundheitliche Versorgung profitiert, der Patient wird als Kunde verstanden, seine präventive Mitwirkungspflicht steigt.

Das algorithmische Bewerten von Symptome wird nicht mit den Aufgaben des Arztes konkurrieren, es wird als sinnvolle Ergänzung Bestandteil innerhalb der Gesundheitsversorgung 2040 sein. Auch hier sind Anfänge bereits heutzutage wahrnehmbar: Seltene Krankheitsbilder werden zu Teilen in fachspezifischen Datenbanken identifiziert, systemseitige Empfehlungen für Behandlungsansätze nehmen Einfluss auf die Handlungsweise der am Patienten tätigen Akteure. Die Rechenkapazität von Computern wird die Geschwindigkeit dieser Entwicklung bestimmen.

Trotz allen Fortschritts werden auch im Jahr 2040 bekannte Krankheitsbilder bleiben, neue sogar entstehen. Das Gesundheitsbewusstsein selbst wird sich jedoch massiv verändern, die Selbstverantwortung steigt. Die Fokussierung auf die Prävention zusammen mit Einsatz neuer Technologien wird das Gesundheitswesen revolutionieren. Wachsende Gesundheitsausgaben durch Einsatz neuer Technologien werden unvermeidbar sein. Sinkende Behandlungsfälle, kürzere Behandlungszeiten sowie der bewusstere Umgang mit der eigenen Gesundheit reduzieren wiederum die Gesundheitsausgaben und bilden somit ein Gleichgewicht. Entwicklungen von patientenindividuellen Behandlungsmethoden werden in den Vordergrund rücken. Nanotechnologie hat die Möglichkeit, intelligente Medikamente oder aus heutiger Sicht revolutionäre neue Ansätze zum Beispiel bei nichtinvasiven Behandlungen zum Einsatz zu bringen. Dagegen wird die Herstellung innerer Organe aus eigenen Stammzellen auch im Jahr 2040 ein Zukunftstraum bleiben.

Körperliche und geistige Fitness und somit die Achtsamkeit auf das eigene Empfinden kann eine zentrale Rolle einnehmen. Die positive Selbst- bzw. Körperwahrnehmung ist eine Folge dessen. Sie wird nicht zur absoluten Selbstoptimierung dienen sondern mit einer Entschleunigung zu einem neuen, gesunden Gleichgewicht führen. Der gesundheitliche Check-up „to go“ befindet sich bereits heute im Einsatz. Sensoren, angebracht am Körper, messen und vergleichen Parameter, um via App eine patientenindividuelle Handlungsempfehlung darzustellen. Das Einarbeiten dieser Sensoren in Kleidungsstücken ist gleichfalls bereits heute Realität. Ein Resultat: Die Diagnostik erhält Einzug in das private Umfeld.

Neue medizinisch-technische Angebote werden mit verantwortbaren Kosten und auf Basis ethischer Grundprinzipien das sinnvolle Miteinander von Mensch, Technik und Digitalisierung darstellen. In sinnvoller Symbiose führen sie zur Weiterentwicklung unseres Gesundheitssystems und können somit zur Grundlage des Ausbaus unserer eigenen Gesundheit werden. Aber eines muss garantiert sein: Die Lösungen müssen stets einfach und für jeden Menschen verständlich ausfallen. Erst dann ist eine flächendeckende Bereitschaft zur Annahme vorstellbar. 